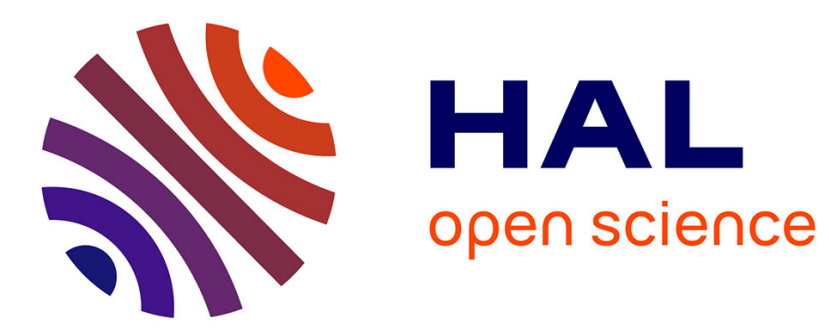

\title{
Estimating the attraction domain for the boost inverter
} Carolina Albea-Sanchez, Francisco Gordillo

\section{To cite this version:}

Carolina Albea-Sanchez, Francisco Gordillo. Estimating the attraction domain for the boost inverter. Asian Journal of Control, 2012, 15 (1), pp.169-176. 10.1002/asjc.519 . hal-00750720

\section{HAL Id: hal-00750720 https://hal.science/hal-00750720}

Submitted on 12 Nov 2012

HAL is a multi-disciplinary open access archive for the deposit and dissemination of scientific research documents, whether they are published or not. The documents may come from teaching and research institutions in France or abroad, or from public or private research centers.
L'archive ouverte pluridisciplinaire HAL, est destinée au dépôt et à la diffusion de documents scientifiques de niveau recherche, publiés ou non, émanant des établissements d'enseignement et de recherche français ou étrangers, des laboratoires publics ou privés. 


\title{
Estimating the attraction domain for the boost inverter
}

\author{
C. Albea * $\quad$ F. Gordillo ${ }^{\dagger}$
}

\begin{abstract}
This work presents an approach for estimating the domain of attraction for polynomial systems with state and control-signal constraints including saturation. In many problems, it is possible to derive global stability properties for such systems, neglecting constraints. Consideration of the constraints usually makes the problem much more involved. In this paper, the stability analysis performed for the unconstrained case is used for the problem as a whole. For application of the method, there are powerful computational tools that can be employed in cases of polynomial systems. The technique is not only valid for the analysis of equilibrium points but also for other attractors, such as limit cycles.

As examples, the domain of attraction for given control laws is estimated for a nonlinear DC-DC boost converter as well as for a boost inverter.
\end{abstract}

\section{Introduction}

Many control designs do not take into account system constraints such as actuator saturation and forbidden regions in the state space, in spite of the fact that these kinds of constraints are present in almost any control application. Nonlinear control books contain an abundance of stability analysis examples in which constraints are not present $[13,28]$. The behaviour of the closed-loop constrained system is not necessarily greatly degraded with respect to the case of unconstrained analysis, making this approach very successful. Nevertheless, in some situations the global stability character may be lost, resulting in local stability of the desired operating behaviour within a bounded region of attraction $[15,2]$. Estimation of this region is desirable but represents a difficult problem.

${ }^{*}$ This work has been conducted while C. Albea was with INPG, Gipsa-lab Grenoble, France and the Dpto. de Ing. de Sistemas y Automática, Sevilla, Spain. Now, C. Albea is with both CNRS; LAAS; 7 avenue du colonel Roche, F-31077 Toulouse, France and Université de Toulouse; UPS, INSA, INP, ISAE; UT1, UTM, LAAS; F-31077 Toulouse, France calbea@laas.fr

${ }^{\dagger}$ F. Gordillo is with the Dpto. de Ing. de Sistemas y Automática, Sevilla, Spain gordillo@esi.us.es 
There exist many published methods for estimating the region of attraction (see, for example $[10,13]$ and the references therein). One example of this kind of method is based on Lyapunov theory, in which closed Lyapunov-function level surfaces are utilized to determine approximate sort of (conservative) estimations for the region of attraction [13]. These methods often employ polynomial systems $[14,22,25,26]$. There exist powerful mathematical tools that can be used in the computation of the maximum acceptable level for polynomial systems $[12,18,27,7]$. Some of these tools could be further developed for application to non-polynomial systems as well [6]. Application of these methods would imply to seek for Lyapunov functions in order to be able to deal with the constraints. The search for a Lyapunov function by means of the numerical estimation method may be seen as an advantage as the user would not be required to propose a Lyapunov function. In cases like these, however, the computational methods are faced with a problem that is very difficult to tackle alone and would encounter difficulty in resolving problems in moderately complex systems.

Furthermore, saturation-like functions, which are one of the most common nonlinearities in practice, usually fall outside of the scope of these techniques. Saturations-like functions are typically only considered in the case of linear systems $[1,4,17,19]$, or in specific cases, as for instance in a port-controlled Hamiltonian system [29].

This paper presents a simple idea that solves the problem of estimation of attraction domain estimation for polynomial non-linear systems (among others) with saturation-like constraints and state constraints. The term saturationlike constraints is used for non-linear functions $\gamma(u)$ that appear in the system model and they become the identity, $\gamma(u)=u$, in certain regions of the state space that present the desired behaviour, (those regions are referred to regions in which such constraints are not active). Functions of this sort include typical control signal saturation as well as others, such as rate limiters, for example. Other constraints on the state variables can be considered as well, with an eye to being able to exploit the unconstrained global stability analysis and use the result to obtain a (conservative) estimation of the region of attraction for the constrained case. Employing the Lyapunov function, which is an integral part of this analysis, eliminates the need to search for a Lyapunov function to estimate the domain of attraction, thus simplifying the problem. On the other hand, the estimated attraction region is included in the domain where the saturation-like constraints are not active and, therefore, the method introduces a new source of conservatism, which could prove to be useful for solving problems when all other methods fail.

To demonstrate the effectiveness of this technique, two electronic application examples are presented. These applications use boost topology in order to obtain oscillating current in such a way that the output voltage can be higher than the input voltage. In the first application, the DC-DC boost converter, physical constraints prevent the output signal from passing through zero, and alternating current is therefore unattainable $[3,9,23]$, leading to a configuration change in the second application in which the circuit is duplicated, thereby yielding an inverter [5]. The control laws analyzed are presented in $[15,11]$ for the first case 
and [2] for the second case. Application of these control laws does not achieve global stability due to two reasons: firstly, the ideal control signal cannot be implemented globally due to control signal saturation; and secondly, the circuit imposes physical constraints on certain state variables: the capacitor voltages, for example, cannot be negative. These reasons motivate the computation of an estimated attraction domain, in such a way that in the starting phase, the system must be driven into a point inside this region. The application of the method produces good, albeit conservative, results. However, certain difficulties should be mentioned here: 1) the system equations are quite involved and can even present non-polynomial terms, namely rational functions; 2) the desired attractor is not an equilibrium point but a limit cycle. Both complications make the use of any other analysis method a formidable task.

The rest of this article is organized as follows: in Section 2 the problem statement as well as the proposed method for estimation of the attraction domain are presented. Section 3 will provide an overview of sums of squares optimization, the numerical tool that is employed in the application of the proposed technique. Section 4 is devoted to the practical cases. Conclusions are drawn in Section 5.

\section{Estimation of the attraction domain}

In every control system, the control signal is subject to physical constraints such as saturations, rate-limiters, etc. As for control designs, such constraints are typically disregarded and the resulting control law is applied to the actuator. In this way, if the designed control law is $u_{d}=\alpha(x)$, where $x$ is the state variable, the actual control signal is $u=\gamma\left(u_{d}\right)=\gamma(\alpha(x))$, where $\gamma(\cdot)$ is a saturation-like function. This approach is valid when the actual expression for $u$ is used in the stability analysis of the resultant system. It is however quite common to neglect the actuator constraints in the stability analysis so as to simplify the analysis. In fact, the local stability property is not usually affected by these constraints, since in a neighborhood $\mathcal{D}$ of the desired attractor they are not active, that is, $\gamma(\alpha(x))=\alpha(x) \forall x \in \mathcal{D}$. However, the resulting attraction domain may be affected by constraints $\gamma$. This study deals with the estimation of this attraction domain based on a stability analysis that neglects the constraints.

The analysis can also take into account state variable constraints in the following sense. Assume that there exists a "forbidden" region in the state space. This means that the system state must remain within the boundaries of a pre-specified admissible ("safe") region. The estimation of the domain of attraction should take into account these constraints.

Formally, the problem can be stated as follows:

Actual system Consider a control system type defined as such:

$$
\dot{x}=f_{a}(x, u),
$$

where $x \in \mathcal{S} \subset \mathbb{R}^{n}, u \in \mathbb{R}^{n_{u}}$. Function $f_{a}$ may include saturation-like functions. Furthermore, due to physical considerations, the state of the system must not go out of an admissible region $\mathcal{T}$. 
Unconstrained system Assume that an approximate model of the system is

$$
\dot{x}=f(x, u),
$$

where function $f: \mathbb{R}^{n} \rightarrow \mathbb{R}^{n}$ and in $\mathcal{D}, f(x, u) \equiv f_{a}(x, u)$. The reader should assume that this approximate model includes neither the saturation-like functions nor the state constraints.

Assume that a control law $u=\alpha(x)$ has been designed for the unconstrained model (2) for a given control objective.

Remark 1 The control objective is not necessarily the stabilization of an equilibrium point, but perhaps the stabilization of limit cycles, for instance, as seen in the examples in Section 4, can be considered.

Assumption 1 There is a widely known radially unbounded Lyapunov function $V(x)$, in which a compact positively invariant set $\Omega, \frac{\partial V}{\partial x} f(x, \alpha(x)) \leq 0$. Let $\mathcal{M}$ be the largest invariant subset of the set for which $\dot{V}=0$ in $\Omega$.

By the LaSalle invariance principle, Assumption 1 guarantees that the trajectories of the unconstrained model tend to $\mathcal{M}$. It is implicitly assumed that this is the desired behaviour. Notice that if the original Lyapunov theorem is used to prove global stability, the previous assumption is also fulfilled.

Assumption 1 also guarantees local stability for system (1). The problem lies in the estimation of the domain of attraction.

The key is to ensure that the system state remains within the boundaries of the region where saturations are not active, thus introducing new constraints. A conservative estimation of the region of attraction can then be easily obtained. The idea of the method is schematically shown in Fig. 1. The advantage of the relative ease with which the estimation is obtained, however, is compromised by the fact that it may be far too conservative. Nevertheless, in many problems this simple idea may give satisfactory results.

Assumption 2 Consider system (1) with control law $u=\alpha(x)$. Let be $\mathcal{A} \triangleq$ $\mathcal{S} \cap \mathcal{T}$, that is, the intersection between the safe region and the region where the saturation-like functions are not active. It is assumed that this set can be estimated by a set of inequalities $g(x) \geq 0$, where $g: \mathbb{R}^{n} \rightarrow \mathbb{R}^{n_{g}}$.

Now the problem can be transformed as follows:

Given a control system $\dot{x}=f(x, u)$ with constraints in both the state variables and the control input $g(x) \geq 0$, assume that a control law $u=\alpha(x)$ has been designed such that global stability is confirmed when no constraints are taken into account. The problem lies in estimating a region of attraction for the real system with constraints when this control law is applied. 


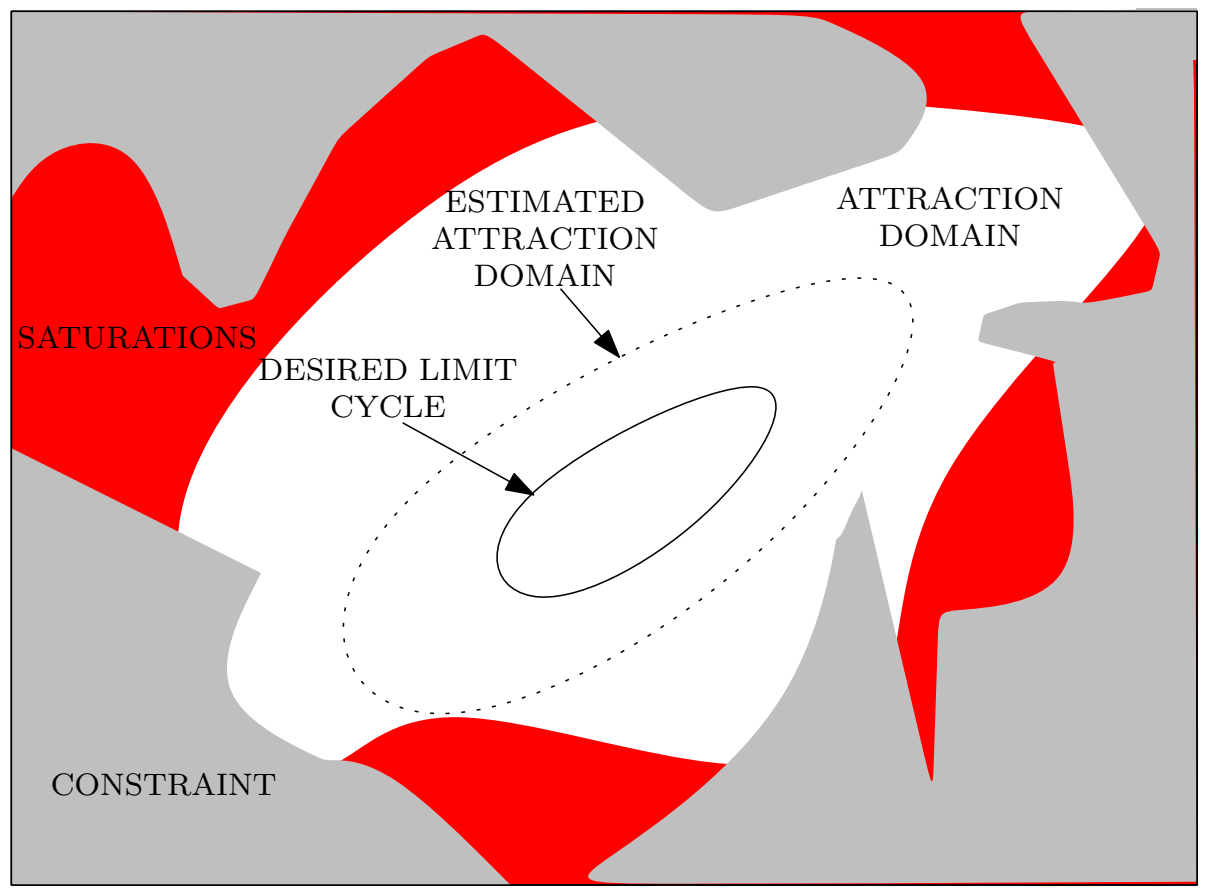

Figure 1: Estimated attraction domain where the constraints are not active. 
A (conservative) estimation for the attraction domain of the system with constraints is given by the following theorem:

Theorem 1 Under assumptions 1 and 2, assume that there exists a constant $c>0$ such that in the set $\Omega_{c}=\{x: V(x) \leq c\}$, the constraints $g(x) \geq 0$ are satisfied. Then, all trajectories of the system with constraints starting at $\Omega_{c}$ tend to $\mathcal{M} \cap \Omega_{c}$.

Proof Since in $\Omega_{c}$ the constraints are satisfied, the results for the unconstrained system are valid in $\Omega_{c}$. Therefore, $\dot{V} \leq 0$ in $\Omega_{c}$ and $\Omega_{c}$ is positively invariant. Furthermore, since $V(x)$ is radially unbounded $\Omega_{c}$ is compact. The statement can be validated by applying LaSalle's invariance principle.

Remark 2 Since $\mathcal{M} \cap \Omega_{c} \subset \mathcal{M}$, the theorem guarantees the desired asymptotic behaviour for the system with constraints.

Remark 3 As with other techniques for estimation of attraction domain, the present method is conservative. In this case the conservativeness is mainly due to two facts:

- The estimation of the region of attraction is restricted to domains in which $V \leq c$.

- The method considers the saturation-like functions as hard constraints. Nevertheless, there may be points where the saturations are active in the actual attraction domain.

Using Theorem 1, the problem is reduced to finding a value $c>0$ such that $g(x) \geq 0$ at the points where $V(x) \leq c$. In order to use numerical tools for the determination of $c$, as will be seen in the next sections, the optimization problem can be stated as follows:

Problem 1 Maximize $c$

subject to:

$$
(V(x)-c)+p_{i}(x) g_{i}(x)-\varepsilon_{i} \geq 0 \quad i=1, \ldots, N,
$$

where $p_{i}(x)$ are unknown semi-definite positive functions and $\varepsilon_{i}>0 ; \quad i=$ $1, \ldots, N$. The purpose of constraint (3) is to validate the Theorem 1 hypothesis. To observe this, notice that at the boundary of the set $\Omega_{c}, V(x)=c$ and, thus, the above constraints are reduced to $p_{i}(x) g_{i}(x) \geq \varepsilon_{i}>0$. As functions $p_{i} \geq 0$, the constraints $g_{i}(x) \geq 0$ are satisfied at the points on the boundary of $\Omega_{c}$. Furthermore, in the interior of this set, $V(x)-c<0$ and, thus, these constraints are also satisfied. The $p_{i}$ functions lend even more degrees of freedom, thereby increasing problem feasibility. The small $\varepsilon_{i}$ constants are pre-specified and are necessary in order to avoid problems at the points where $p_{i}(x)=0$. The introduction of $\varepsilon_{i}$ parameters constitute a new source of conservatism.

Remark 4 Notice that the expression of $\dot{V}$ is not considered in the optimization Problem 1. This fact may greatly reduce the complexity of the numerical problem. 
In this work, sum-of-squares optimization is used order to solve this problem. For this, a new assumption is needed.

Assumption 3 Functions $f(x, u)$ and $g(x)$ are polynomial.

\section{Sum of squares optimization}

Sum of squares optimization is an optimization technique based on the Sum Of Squares (SOS) decomposition for multivariate polynomials. A multivariate polynomial $p(x)$ is said to be an SOS, if there exist polynomials $f_{1}(x), \ldots, f_{m}(x)$, such that:

$$
p(x)=\sum_{i=1}^{m} f_{i}^{2}(x)
$$

and therefore, $p(x) \geq 0[21]$.

An SOS program has the following form [21]:

Minimize the linear objective function

$$
w^{T} c,
$$

where $c$ is a vector formed from the (unknown) coefficients of:

- polynomials $p_{i}(x)$, for $i=1,2, \ldots, N_{1}$

- sum of squares $p_{i}(x)$, for $i=N_{1}+1, \ldots, N_{2}$

such that:

$$
\begin{aligned}
& g_{0, j}(x)+\sum_{i=1}^{N} p_{i}(x) g_{i, j}(x)=0 \\
& \quad \text { for } j=1,2, \ldots, M_{1} . \\
& g_{0, j}(x)+\sum_{i=1}^{N} p_{i}(x) g_{i, j}(x) \text { are SOS }, \\
& \quad \text { for } j=M_{1}+1, \ldots, M_{2} .
\end{aligned}
$$

where $w$ is the linear objective function weighting coefficients vector, and $g_{i, j}(x)$ represent certain scalar constant coefficient polynomials.

Currently, SOS programs are solved by reformulating them as semi-definite programs (SDPs), which in turn are solved efficiently, e.g., using interior point methods. Several commercial as well as non-commercial software packages are available for solving SDPs. SOSTOOLS [20] is a Matlab toolbox that performs this conversion automatically, calls the SDP solver, and converts the SDP solution back to that of the solution of the original problem.

The problem stated in the previous section can be addressed solving the following SOS problem: 
Problem 2 Maximize $c$

subject to:

$$
\begin{aligned}
& (V(x)-c)+p_{i}(x) g_{i}(x)-\varepsilon_{i} \\
& \text { are } S O S ; \quad i=1, \ldots, N,
\end{aligned}
$$

where $p_{i}$ are unknown SOS polynomials. This problem is more restricted than that presented in the previous section. Nevertheless, any solution to SOS problem 2 is a solution to problem 1 .

Remark 5 Assumption 3 can be relaxed since other types of functions, such as trigonometric functions [16] or rational functions (e.g., the application examples in the next section) can be considered.

\section{Some practical cases: electronic converters}

The method developed above is applied to two practical cases: a DC-DC boost converter and a boost inverter. The control laws used for these systems appear in [15] and [2] respectively where the global stability analysis is performed neglecting saturations and forbidden regions.

\subsection{Application 1: Oscillations in a DC-DC boost con- verter}

A boost circuit is usually used as a DC-DC converter and is of particular interest due to the fact that it generates an output voltage larger than its input voltage. Nevertheless, in $[9,8]$ it is proposed to convert DC voltage into an oscillating one. However, alternating current cannot be generated with this circuit, as the output current cannot change its sign. In this section the attraction domain is analyzed when this converter is controlled with the law presented in [15]. For completeness, the design is summarized here.

The normalized model of the DC-DC boost converter is:

$$
\begin{aligned}
& \dot{x}_{1}=-u x_{2}+1 \\
& \dot{x}_{2}=u x_{1}-a x_{2}
\end{aligned}
$$

where $x_{1}$ is the normalized current and $x_{2}$ is the normalized capacitor voltage defining the system states and where $a$ is a constant and $u$ is the control input, which has a direct relationship with the average duty-cycle. System (5)-(6) can therefore be assumed to be a continuous system [15].

Under the assumption that $a$ is a known constant, a nonlinear control law based on the Hamiltonian approach is proposed in [15]. The design is based on the following change of coordinates:

$$
\begin{aligned}
\zeta_{1} & =\frac{x_{1}^{2}+x_{2}^{2}}{2} \\
\zeta_{2} & =x_{1}-a x_{2}^{2}+\zeta_{20}
\end{aligned}
$$


The aim of the control design is to ensure that the following function:

$$
\Gamma=\omega^{2}\left(\zeta_{1}-\zeta_{10}\right)^{2}+\left(\zeta_{2}-\zeta_{20}\right)^{2}-\mu
$$

tends to zero. This goal corresponds to an ellipse in the current-voltage plane and is assumed that correspond to the desired behaviour. Notice that this desired behaviour is a limit cycle. Based on this definition, the nonlinear control law proposed in [15] can be expressed as follows:

$$
u=\frac{1+2 a^{2} x_{2}^{2}+k \Gamma\left(\zeta_{2}-\zeta_{20}\right)+\omega^{2}\left(\zeta_{1}-\zeta_{10}\right)}{x_{2}\left(1+2 a x_{1}\right)}
$$

where $k>0$ defines the speed of the transient response.

In [15] it is proven that, with this control law, for all initial conditions except the origin, the trajectories of the system tend to the curve $\Gamma=0$. Nevertheless, there are several constraints in the state variable that make this analysis useless from the practical point of view. These constraints are of several types:

C1. Constraints $0 \leq u \leq 1$ make control law (7) unfeasible in the full state space. In practice, $u$ is chosen equal to 1 when control (7) is greater than 1 and, $u$ is chosen equal to zero when control (7) is negative. Therefore, this constraint is 'soft' in the sense that if the system arrives at a point where the constraints are violated, the analysis of [15] is no longer valid for the system with constraints. The point may nonetheless still lie inside the attraction domain of the desired limit cycle. Therefore, these constraints are saturation-like.

C2. Capacitor voltage cannot be negative in this circuit, which implies $x_{2} \geq 0$. This is considered to be a 'hard' constraint, meaning that such a situation should always be avoided.

C3. Finally, the control law is not feasible when the denominator in (7) is zero. This constraint is actually contained in $\mathrm{C} 1$, since when the denominator is close to zero the control signal assumes large (positive or negative) values.

The objective of this work is to obtain a (possibly conservative) estimation for the region of attraction of the resultant system taking these physical constraints into account.

The stability proof in [15] for the unconstrained (fictitious) case is based on LaSalle's invariance principle. The Lyapunov function used is:

$$
V=\frac{\Gamma^{2}}{2} \text {. }
$$

The constraints are (only constraints $\mathrm{C} 1$ and $\mathrm{C} 2$ are presented here; constraint C3 will be discussed later):

$$
\begin{aligned}
u(x) & \leq 1 \\
u(x) & \geq 0 \\
x_{2} & \geq 0
\end{aligned}
$$


The expression for $u$, which is given by (7) is not polynomial but rather a rational function. Nevertheless, by expressing it as a quotient $u(x)=n(x) / d(x)$ polynomials, all the constraints can be formulated in standard form. It can then be assumed that polynomial $d(x)$ does not vanish at any point along the objective curve $\Gamma(x)=0$. Otherwise, control law (7) is not valid for this problem. Therefore, the sign of $d(x)$ is constant along $\Gamma(x)=0$ and, through continuity in the neighborhood of this curve. By means of numerical inspection, it can be verified that, for the circuit parameters given below, $d(x)>0$ on $\Gamma(x)=0$. With this consideration in mind, constraints (9)-(11) can be written as polynomial constraints:

- $d(x)-n(x) \geq 0$

- $n \geq 0$

- $x_{2} \geq 0$

Therefore, the problem to be solved is as follows:

$$
\text { Minimize }(-c)
$$

subject to:

$$
\begin{aligned}
& (V(x)-c)+p_{1}(x)(d(x)-n(x))-\varepsilon_{1} \geq 0 \\
& (V(x)-c)+p_{2}(x) n(x)-\varepsilon_{2} \geq 0 \\
& (V(x)-c)+p_{3}(x) x_{2}-\varepsilon_{3} \geq 0 \\
& p_{1}(x), p_{2}(x), p_{3}(x) \text { are SOS }
\end{aligned}
$$

Notice that constraint C3 is considered in the previous set of constraints. Indeed, constraint (13) implies $n(x) \geq \varepsilon_{2}>0$ for $V(x) \leq c$, while constraint (13) implies $d(x) \geq n(x)+\varepsilon_{1}$ for $V(x) \leq c$. This implies that $d(x)>0$ in $V(x) \leq c$.

The following analysis can be directly modified for the case when $d(x)<0$.

\section{Results}

The values of the circuit parameters are taken from [15], resulting in a value for parameter $a$ equal to 0.9045 . The desired output AC voltage of the boost circuit is:

$$
v_{\text {conv }}^{*}=135+15 \sin 2 \pi 50 t
$$

Software SeDuMi [24] was used as the SDP solver under SOSTOOLS. The values for parameters $\varepsilon_{i}$ are chosen equal to $10^{-6}$ while the chosen order for the unknown polynomials $p_{i}$ is 6 . The solution obtained is: $c^{*}=208.5$. This value is close to the optimal value as verified by numerical inspection and thus confirms that for the point $x^{(1)}=\left(\begin{array}{ll}6.9 & 1.1\end{array}\right)^{\top}$, which corresponds to $V\left(x^{(1)}\right)=355$, the control signal $u$ takes a value equal to 1.0001 .

Nevertheless, the result is probably conservative as was pointed out in Remark 3 . 
Figure 2 shows the resultant estimation of the attraction domain. Note, this attraction domain is presented in the voltage-current plane. Also shown in Fig. 2 is the smaller Lyapunov level curve which inscribes the forbidden point given before $\left(x^{(1)}\right)$, as well as the desired limit cycle $\Gamma=0$.

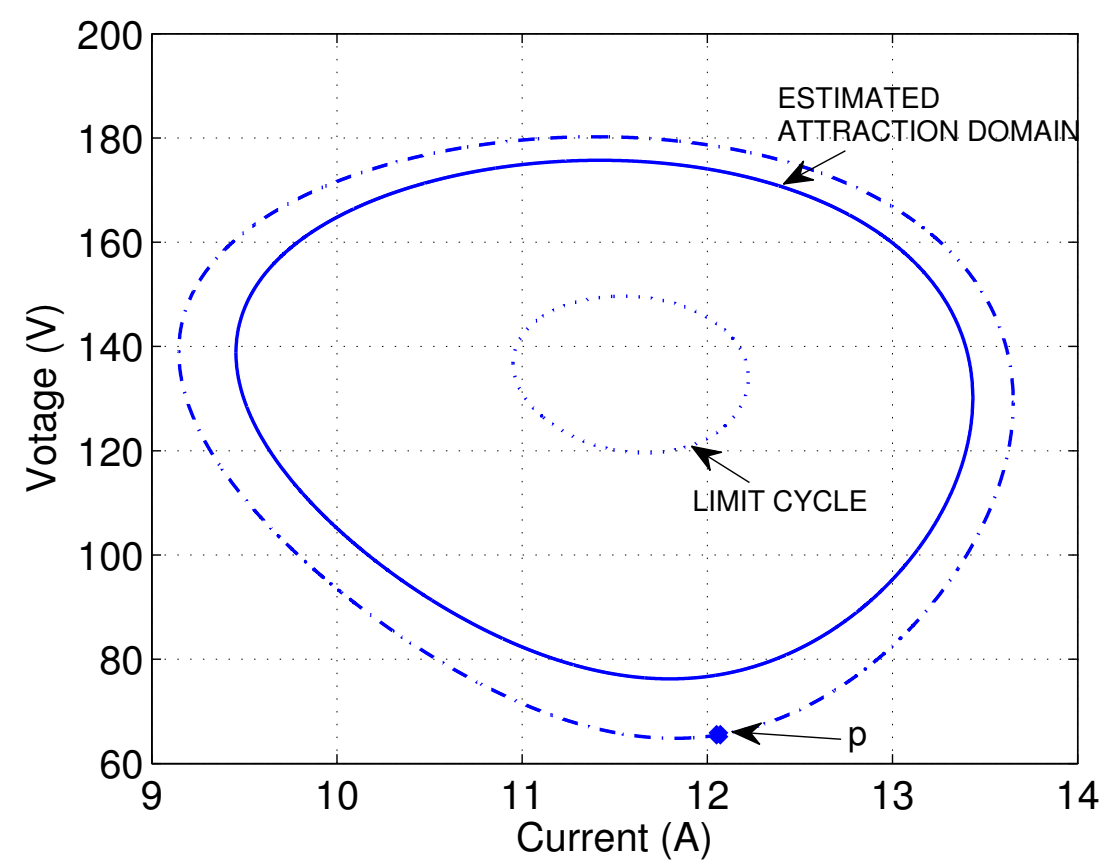

Figure 2: Example 1: Estimated attraction domain (solid); Lyapunov level curve (dashed) corresponding to the a forbidden point $x^{(1)}$; and desired limit cycle (dotted).

Notice that the method proposed in this paper may be valid when the system presents complex fractional equations.

\subsection{Application 2: boost inverter}

A means of obtaining AC output using boost topology a double boost is proposed in [5]. In this section the attraction domain is analyzed when this converter is controlled with the law presented in [2]. The design is summarized here, with the normalized model of this system being:

$$
\begin{aligned}
& \dot{x}_{1}=-u_{1} x_{2}+1 \\
& \dot{x}_{2}=u_{1} x_{1}-a\left(x_{2}-x_{4}\right) \\
& \dot{x}_{3}=-u_{2} x_{4}+1 \\
& \dot{x}_{4}=u_{2} x_{3}+a\left(x_{2}-x_{4}\right)
\end{aligned}
$$


The nature of the constants and variables here are similar to that in the case before: $x_{1}, x_{3}$ are the normalized currents, $x_{2}, x_{4}$ are the normalized capacitor voltages and $u_{1}, u_{2}$ are the control inputs. Note that this system is of greater dimensions than the system described in example 1.

The control law designed in [2] for the unconstrained (fictitious) case employs the following Lyapunov function:

$$
V=\frac{\Gamma_{1}^{2}}{2}+\frac{\Gamma_{2}^{2}}{2}
$$

with

$$
\begin{aligned}
& \Gamma_{1}=\omega^{2}\left(\zeta_{1}-\zeta_{10}\right)^{2}+\left(\zeta_{2}-\zeta_{20}\right)^{2}-\mu \\
& \Gamma_{2}=\omega^{2}\left(\zeta_{3}-\zeta_{30}\right)^{2}+\left(\zeta_{4}-\zeta_{40}\right)^{2}-\mu
\end{aligned}
$$

In [2], it is proved that $\dot{V}<0$ by LaSalle's invariance principle.

The corresponding control laws are

$$
\begin{aligned}
u_{1}= & \frac{1+2 a^{2} x_{2}^{2}-3 a^{2} x_{2} x_{4}+a^{2} x_{4}^{2}+a^{2} x_{2} \dot{x}_{4}}{x_{2}+2 a x_{1} x_{2}-a x_{4} x_{1}} \\
& +\frac{k_{1} \Gamma_{1}\left(\zeta_{2}-\zeta_{20}\right)+\omega^{2}\left(\zeta_{1}-\zeta_{10}\right)}{x_{2}+2 a x_{1} x_{2}-a x_{4} x_{1}} \\
u_{2}= & \frac{1+2 a^{2} x_{4}^{2}-3 a^{2} x_{2} x_{4}+a^{2} x_{2}^{2}+a^{2} x_{4} \dot{x}_{2}}{x_{4}+2 a x_{3} x_{4}-a x_{2} x_{3}} \\
& -\frac{k_{2} \Gamma_{2}\left(\zeta_{4}-\zeta_{40}\right)-\omega^{2}\left(\zeta_{3}-\zeta_{30}\right)}{x_{4}+2 a x_{3} x_{4}-a x_{2} x_{3}}
\end{aligned}
$$

This system has the same constraints as those of the previous application: $\mathrm{C} 1, \mathrm{C} 2$ and $\mathrm{C} 3$. It should be noted that in this case there are two control inputs $\left(u_{i}, \quad i=1,2\right)$ and as before, two capacitor voltages $\left(x_{i}, \quad i=2,4\right)$, and numerical inspection reveals the case in which $d(x)>0$ is the one that should be analyzed. The optimization problem results

$$
\text { Minimize }(-c)
$$

subject to:

$$
\begin{aligned}
& (V(x)-c)+p_{1}(x)\left(n_{1}(x)-d_{1}(x)\right)-\varepsilon_{1} \geq 0 \\
& (V(x)-c)+p_{2}(x)\left(n_{2}(x)-d_{2}(x)\right)-\varepsilon_{2} \geq 0 \\
& (V(x)-c)-p_{3}(x) n_{1}(x)-\varepsilon_{3} \geq 0 \\
& (V(x)-c)-p_{4}(x) n_{2}(x)-\varepsilon_{4} \geq 0 \\
& (V(x)-c)+p_{5}(x) x_{2}-\varepsilon_{5} \geq 0 \\
& (V(x)-c)+p_{6}(x) x_{4}-\varepsilon_{6} \geq 0 \\
& p_{1}(x), p_{2}(x), p_{3}(x), p_{4}(x), p_{5}(x), p_{6}(x) \text { are SOS }
\end{aligned}
$$




\section{Results}

The parameter values are taken from [2]. The desired output voltage is:

$$
v_{i n v}^{*}=40 \sin 2 \pi 50 t
$$

The tuning parameters $\varepsilon_{i}$ are equal to $10^{-6}$ and the degree of the unknown $p_{i}$ polynomials is assigned a value of 3 .

The solution is obtained in approximately ten minutes on a $\mathrm{PC}(1.66 \mathrm{GHz}$ Intel Core2): $c^{*}=1.5$. As a means of corroborating this result, a numerical inspection has found that for a value of $x^{(2)}=\left(\begin{array}{llll}0 & -0.1 & 0.4 & 8.1\end{array}\right)^{\top}$, which corresponds to $V\left(x^{(2)}\right)=2.38$, the constraint $x_{2} \geq 0$ is violated.

\section{Conclusions}

A methodology for the estimation of the region of attraction that takes system physical constraints into consideration has been presented in this article. The kind of problems considered may present a high degree of difficulty due to the model and control law nonlinearities including saturation-like constraints. The method is based on the search for a Lyapunov level surface where the constraints are satisfied. This problem can be written as a sum of squares optimization problem, for which good numerical tools are available. This approach has general applicability to cases where the global stability of the unconstrained problem has been demonstrated (by means of Lyapunov methods) and in which extension to the constrained case is desired. This method is useful even when the degree and complexity of the equations are high. The closed-loop model and constraints must be polynomial or rational (there are cases, nevertheless, where SOS programming has been applied to trigonometric and other terms [16]). Conservativeness of the method has also been discussed.

The usefulness of the method has been shown with two electronic applications.

\section{Acknowledgments}

The authors are grateful to the anonymous reviewers of this paper for their valuable comments and suggestions. This research has been partially supported by the MICINN-FEDER grant DPI2009-09961.

\section{References}

[1] T. Álamo, A. Cepeda, D. Limón, and E.F. Camacho. Estimation of the domain of attraction for saturated discrete-time systems. International Journal of Systems Science, 37(8):575-583, 2006.

[2] C. Albea, F. Gordillo, and J. Aracil. Control of the Boost DC-AC Converter by Energy Shaping. In Proc. IEEE Industrial Electronics (IECON), pages 754-759, 2006. 
[3] D. Biel, E. Fossas, F. Guinjoan, and R. Ramos. Sliding mode control of a boost-buck converter for ac signaltracking task. In Proc. IEEE International Symposium on Circuits and Systems, (ISCAS), 5, 1999.

[4] F. Blanchini and S. Miani. Any domain of attraction for a linear constrained system is a tracking domain of attraction. In Proc. IEEE in the Conference on Decision and Control (CDC), 5:5065-5070, 2000.

[5] RO Cáceres and I. Barbi. A boost DC-AC converter: analysis, design, and experimentation. In Trans. on IEEE Power Electronics, 14(1):134-141, 1999.

[6] G. Chesi. Estimating the domain of attraction for non-polynomial systems via LMI optimizations. Automatica, 45(6):1536-1541, 2009.

[7] G. Chesi, A. Garulli, A. Tesi, and A. Vicino. Solving quadratic distance problems: an LMI-based approach. In Trans. IEEE Automatic Control, 48(2):200-212, 2003.

[8] E. Fossas, J.M. Olm, and H. Sira-Ramírez. Iterative approximation of limit cycles for a class of Abel equations. Physica D: Nonlinear Phenomena, 237(23):3159-3164, 2008.

[9] E. Fossas-Colet and JM Olm-Miras. Asymptotic tracking in DC-to-DC nonlinear power converters. Discrete and Continuous Dynamical Systems Series B, 2(2):295-311, 2002.

[10] R. Genesio, M. Tartaglia, and A. Vicino. On the estimation of asymptotic stability regions: State of the art and new proposals. In Trans. IEEE Automatic Control, 30(8):747-755, 1985.

[11] F. Gordillo, DJ Pagano, and J. Aracil. Autonomous oscillation generation in electronic converters. In Proc. IEEE International Workshop on Electronics and System Analysis (IWESA), 2004.

[12] Birkett Huber and Bernd Sturmfels. A polyhedral method for solving sparse polynomial systems. In Trans Mathematics of Computation, 64(212):1541$1555,1995$.

[13] Hassan K. Khalil. Nonlinear Systems. Prentice Hall, third edition edition, 2002.

[14] A. Levin. An analytical method for estimation the domain of attraction for polynomial differential equations. In Trans. IEEE Automatic Control, pages $2471-2475,1994$.

[15] D. J. Pagano, J. Aracil, and F. Gordillo. Autonomous oscillation generation in the boost converter. In Proc. IFAC World Congress, 2005. 
[16] A. Papachristodoulou and S. Prajna. On the construction of Lyapunov functions using the sum of squares decomposition. In Proc. IEEE Decision and Control (CDC), 3:3482-3487, 2002.

[17] TE Pare, H. Hindi, JP How, and SP Boyd. Synthesizing stability regions for systems with saturating actuators. In Proc. IEEE Conference Decision and Control (CDC), 2:1981-1982, 1998.

[18] Pablo A. Parrilo. Structured Semidefinite Programs and Semialgebraic Geometry Methods in Robustness and Optimization. PhD thesis, California Institute of Technology, Pasadena, California, 2000.

[19] C. Pittet, S. Tarbouriech, and C. Burgat. Stability regions for linear systems with saturating controls viacircle and Popov criteria. In In Proc. IEEE Decision and Control (CDC), volume 5, pages 4518-4523, 1997.

[20] S. Prajna, A. Papachristodoulou, and PA Parrilo. Introducing SOSTOOLS: a general purpose sum of squares programming solver. In Proc. IEEE Conference on Decision and Control (CDC), 1:741-746, 2001.

[21] Stephen Prajna, Antonis Papachristodoulou, Peter Seiler, and Pablo A. Parrilo. SOSTOOLS, Sum of Squares Optimization Toolbox for MATLAB. available from http://www.cds.caltech.edu/sostools, 2004.

[22] B. G. Romanchuk. Computing regions of attraction with polytopes: planar case. Automatica, 32(12):1727-1732, 1996.

[23] H. Sira-Ramírez and MT Prada-Rizzo. A Dynamic Pole Assignment Approach to Stabilization and Tracking in DC-to-DC Full Bridge Power Converters. Control Theory and Advanced Technology, 9(1):247-258, 1993.

[24] J.F. Sturm. Using SeDuMi 1.02, a MATLAB toolbox for optimization over symmetric cones. Optimization Methods and Software, 11-12:625-653, 1999.

[25] B. Tibken and K.F. Dilaver. Computation of subsets of the domain of attraction for polynomial systems. In Proc. IEEE Conference Decision and Control (CDC), 3:2651-2656, 2002.

[26] U. Topcu, A. Packard, P. Seiler, and G. Balas. Help on sos [ask the experts]. IEEE Control Systems Magazine, 30(4):18-23, 2010.

[27] Jan Verschelde. Algorithm 795: Phcpack: a general-purpose solver for polynomial systems by homotopy continuation. In ACM Trans. on Mathematical Software (TOMS), 25(2):251-276, 1999.

[28] M. Vidyasagar. Nonlinear systems analysis. Society for Industrial Mathematics, 2002.

[29] A. Wei, Y. Wang, and X. Hu. Estimate of domain of attraction for a class of port-controlled hamiltonian systems subject to both actuator saturation and disturbances. Asian Journal of Control, 2011. 